\title{
高光热效应碳球的快速制备及肿瘤细胞光热治疗
}

\author{
魏成雄，金 金金，殷培楠，吴承伟，张伟 \\ (大连理工大学 工业装备结构分析国家重点实验室, 工程力学系, 生物与纳米力学实验室, 大连 116024)
}

摘 要: 光热治疗是一种非侵入式的新型肿瘤治疗手段, 可弥补传统治疗方式的不足。碳纳米材料作为一种高效的 光热剂, 在肿瘤光热治疗中表现出巨大的应用潜力。本研究采用超声辅助法使邻苯三酚与甲醛 $5 \mathrm{~min}$ 快速聚合, 经 煅烧处理制备了单分散、粒径均一的碳球。该碳球兼具优良的细胞生物相容性和高光热转换效率。在 $808 \mathrm{~nm}$ 近红 外光照射下, 碳球呈现良好的光热效应和光热稳定性, 光热转换效率达到 $41.4 \%$ 。细胞实验表明, 碳球无明显细胞 毒性，对肿瘤细胞具有显著的光热杀伤效果。制备的高光热效应碳球光热剂有望用于肿瘤光热治疗。

关 键 词: 超声辅助法; 碳球; 光热治疗; 肿瘤

中图分类号: TB34 文献标志码: A

\section{Carbon Spheres for Photothermal Therapy of Tumor Cells: Rapid Preparation and High Photothermal Effect}

\author{
WEI Chengxiong, JIN Xin, YIN Peinan, WU Chengwei, ZHANG Wei
}

\begin{abstract}
(Laboratory of Biology and Nanomechanics, Department of Engineering Mechanics, State Key Laboratory of Structural Analysis for Industrial Equipment, Dalian University of Technology, Dalian 116024, China)
\end{abstract}

\begin{abstract}
Photothermal therapy is a new non-invasive treatment method, which can make up for the deficiency of traditional tumor therapy. As an efficient photothermal agent, carbon nanomaterials show great potential in the photothermal therapy of tumor. In this study, a rapid polymerization of pyrogallic acid and formaldehyde was induced by ultrasound-assisted method. Carbon spheres with good biocompatibility and high photothermal conversion efficiency were quickly prepared by subsequent calcination treatment. The prepared carbon spheres are highly monodisperse and uniform in size. Under $808 \mathrm{~nm}$ near-infrared light irradiation, they exhibit good photothermal effect and photothermal stability with photothermal conversion efficiency up to $41.4 \%$. Cell experiments show that carbon spheres have no obvious cytotoxicity and can effectively kill the tumor cells under near-infrared light irradiation. This work is expected to construct efficient photothermal carbon spheres for photothermal therapy of tumor.
\end{abstract}

Key words: ultrasound-assisted method; carbon sphere; photothermal therapy; tumor

目前, 癌症的临床治疗方法主要包括手术治疗、 化学治疗和放射治疗。然而, 这些治疗方法均存在 一定的局限性。手术治疗不能确保完全切除肿瘤组
织, 而导致肿瘤复发。化学治疗和放射治疗通常会 伴随严重的副作用 ${ }^{[1-2]}$ 。因此, 迫切需要寻求新的肿 瘤治疗方法来弥补传统治疗方式存在的不足。肿瘤

收稿日期: 2021-03-01; 收到修改稿日期: 2021-03-29

基金项目：国家重点研发计划项目（2018YFA0704103,2018YFA0704104）; 辽宁省自然科学基金项目（2019-KF-02-01）; 中 央高校基本科研业务费项目（DUT20YG129）

National Key Research and Development Program (2018YFA0704103, 2018YFA0704104); Natural Science Foundation of Liaoning Province (2019-KF-02-01); Fundamental Research Funds for the Central Universities (DUT20YG129)

作者简介: 魏成雄（1992-）, 男, 博士研究生. E-mail: weicx@mail.dlut.edu.cn

WEI Chengxiong (1992-), male, PhD. candidate. E-mail: weicx@mail.dlut.edu.cn

通信作者: 张 伟, 教授, E-mail: wei.zhang@dlut.edu.cn

ZHANG Wei, professor. E-mail: wei.zhang@dlut.edu.cn 
光热治疗 (Photothermal therapy, PTT) 作为一种新 型的绿色治疗方法, 近年来已吸引大量科学家从事 相关研究 ${ }^{[3-6]}$ 。由于生物组织在近红外光区域 (700 950 nm) 具有较低的吸收, 近红外光可以较 好地穿透皮肤组织 ${ }^{[7]}$ 。因此, 光热治疗是一种非侵入 式的治疗手段, 可利用近红外光诱导肿瘤部位的光 热剂产生热量实现局部热疗, 从而高效地消融肿瘤 组织, 最大限度减少对正常组织的损伤 ${ }^{[8-9]}$ 。

光热剂性能对于光热治疗效果起决定性作用, 目前, 用于肿瘤光热治疗的纳米光热剂主要分为无 机光热剂 ${ }^{[10]}$ 和有机光热剂[11]。有机光热剂虽然具有 良好的生物相容性, 但其光吸收峰较窄、制备流程 复杂、光热稳定性较差, 限制了其应用。无机光热 剂一般包括金属纳米材料 [12-13]、碳纳米材料 [14-16]、 以及无机化合物纳米材料 [17-19], 由于无机光热剂具 有较高的光热转换效率和良好的光热稳定性, 得到 了广泛研究。尤其是碳纳米材料, 诸如碳纳米管 ${ }^{[20-}$

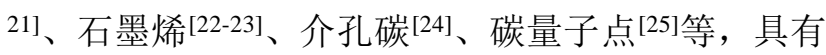
多维度、易于功能化、可作微纳尺度结构设计等优 点, 在肿瘤光热治疗上具有广阔的应用前景。然而, 制备工艺复杂、生物相容性不够理想等限制了碳纳 米材料在肿瘤光热治疗方面的应用。因此, 实现碳 材料的快速制备, 开发兼具良好生物相容性及优异 光热性能的碳基光热剂具有重要意义。

本研究基于超声辅助法实现了高光热效应碳球 的快速制备。该方法使用邻苯三酚和甲醛作为反应 前驱体, 经超声诱导酚醛聚合, 实现 $5 \mathrm{~min}$ 快速制 备酚醛树脂微球, 经烊烧处理最终得到单分散、粒 径均一的碳球。所制备的碳球具有良好的光热性能 和光热稳定性, 表现出较好的细胞生物相容性, 其 诱导的光热效应能够有效杀伤肿瘤细胞, 作为一种 高效的碳基光热剂, 有望被应用于肿瘤的临床光热 治疗。

\section{1 实验方法}

\section{1 试剂与设备}

邻苯三酚（Pyrogallic acid, AR）购自上海阿拉 丁生化科技股份有限公司; 甲醛溶液 (Formaldehyde solution, AR, 37\%) 购自上海麦克林生化科技有限 公司; 氨水 (Ammonia solution, AR, 25\% 28\%) 购自天津科密欧化学试剂有限公司; 无水乙醇

（Ethanol absolute，AR）购自天津富宇精细化工有 限公司; CCK-8 检测试剂盒购自美国 APExBIO 公
司; Calcein-AM 和 PI 检测试剂盒购自江苏凯基生 物技术股份有限公司。超声仪 (VCY-1500, 功率: $1500 \mathrm{~W}$ ) 购自上海研永超声设备有限公司; 近红外 激光器 (MDL-H-808-5W, 波长: $808 \mathrm{~nm}$ ) 购自长春 新产业光电技术有限公司。

\section{2 碳球的制备}

将 $40 \mathrm{~mL}$ 乙醇、 $0.5 \mathrm{~mL}$ 氨水和 $100 \mathrm{~mL}$ 去离子 水混合到 $200 \mathrm{~mL}$ 烧杯中, 搅拌均匀。称取 $1 \mathrm{~g}$ 邻苯 三酚加入到上述溶液中, 分别按邻苯三酚和甲醛摩 尔比为 $2: 1 、 1: 1 、 1: 2$ 和 $1: 4$ 加入甲醛溶液。将上述 混合溶液超声反应 $5 \mathrm{~min}$ 。溶液由浅黄色透明液体变 成黄色悬浮液, 停止超声处理, 通过抽滤分离产物, 用酒精和去离子水分别清洗 3 次, 将所得产物置于 $50{ }^{\circ} \mathrm{C}$ 下真空干燥, 得到淡黄色酚醛树脂粉末。将粉 末置于管式炉中, 在 $\mathrm{N}_{2}$ 保护下 $500{ }^{\circ} \mathrm{C}$ 炦烧 $3 \mathrm{~h}$, 升 温速率为 $5{ }^{\circ} \mathrm{C} / \mathrm{min}$, 煅烧结束后, 自然冷却得到最 终产物。

\section{3 材料表征}

使用扫描电镜 (SEM, Quanta 200, 美国 FEI 公 司) 和透射电镜 (TEM, JEM-2100, 日本电子株式 会社) 观察炦烧前后酚醛树脂纳米球的粒径和形貌; 使用傅里叶红外光谱仪 (FT-IR, Nicolet 6700, 美国 ThermoFisher 公司) 分析样品化学官能团; 使用拉 曼光谱仪 (DXR Microscope, 美国 ThermoFisher 公 司) 分析样品化学结构; 使用紫外可见光光度计 (UV-1800, 日本岛津公司) 测试样品的吸光度; 使 用粒度分析仪 (Zetasizer Nano ZS90, 英国 Malvern 公司) 测试样品的 Zeta 电位和粒径分布; 使用热重 分析仪 (TGAQ500, 美国 TA 公司) 测试样品的热 重曲线。

\section{4 光热性能评价}

将碳球均匀分散到去离子水中, 超声处理 $5 \mathrm{~min}$ 。 取 $0.2 \mathrm{~mL}$ 的碳球悬浮液加入到 $1.5 \mathrm{~mL}$ 的离心管中, 使用 $808 \mathrm{~nm}$ 近红外光照射悬浮液, 照射距离为 10 $\mathrm{cm}$, 使用红外成像仪监测样品温度。改变碳球悬浮 液浓度和激光功率密度, 测试碳球的光热效应。

通过测试碳球悬浮液在近红外光照射下的升温 和自然降温过程, 计算碳球的光热转换效率。取 0.2 $\mathrm{mL}$ 浓度为 $100 \mu \mathrm{g} / \mathrm{mL}$ 的碳球悬浮液放入 $1.5 \mathrm{~mL}$ 的 离心管中, 使用功率密度为 $0.8 \mathrm{~W} / \mathrm{cm}^{2}$ 的近红外光 照射样品, 照射 $8 \mathrm{~min}$ 后, 系统温度达到平衡, 关 
闭激光器, 使样品自然冷却, 整个升温和降温过程 使用红外成像仪监测样品温度。

光热转换效率计算公式如下 ${ }^{[26]}$ :

$$
\sum_{i} m_{i} C_{i} \frac{d T}{d t}=Q_{\mathrm{p}}+Q_{\mathrm{w}}+Q_{\mathrm{l}}
$$

公式 (1) 表示系统的能量平衡方程。式中, $m_{i}$ 和 $C_{i}$ 分别为悬浮液中水和碳球的质量及比热容, $T$ 为悬浮液的温度, $t$ 为近红外光照射时间, $Q_{\mathrm{p}}$ 为碳 球光热转换产生的热量, $Q_{\mathrm{w}}$ 为水光热转换产生的热 量, $Q_{1}$ 为系统流向周围环境的热量。

$$
Q_{\mathrm{p}}=I\left(1-10^{-A_{808}}\right) \eta
$$

式中, $I$ 为输入系统的激光功率, $A_{808}$ 为碳球在波长 为 $808 \mathrm{~nm}$ 处的吸光度, $\eta$ 为碳球的光热转换效率。

$$
Q_{1}=h S \Delta T
$$

式中, $h$ 为传热系数, $S$ 为离心管的表面积, $\Delta T=T$ $T_{\text {surr }}, T$ 为系统的实时温度, $T_{\text {surr }}$ 为环境温度。

当系统温度达到平衡时, 光热转换产生的热量 等于耗散的热量, 此时:

$$
Q_{\mathrm{p}}+Q_{\mathrm{w}}=Q_{\mathrm{l}}=h S \Delta T_{\text {max }}
$$

式中, $\Delta T_{\max }$ 为系统处于平衡状态时的温度变化值, 由公式 (2 4) 可以得到光热转换效率的计算公式:

$$
\eta=\frac{h S \Delta T_{\max }-Q_{\mathrm{w}}}{I\left(1-10^{\left.-A_{808}\right)}\right.}
$$

式中, 参数 $h S$ 未知, 引入 $\theta$ 求 $h S, \theta$ 定义为:

$$
\theta=\frac{\Delta T}{\Delta T_{\max }}
$$

将公式 (3) 和 (6) 带入公式 (1) 中, 可推导 得到如下公式:

$$
\frac{\mathrm{d} \theta}{\mathrm{d} t}=\frac{h S}{\sum_{i} m_{i} C_{i}}\left(\frac{Q_{\mathrm{p}}+Q_{\mathrm{w}}}{h S \Delta T_{\max }}-\theta\right)
$$

当激光关闭后, 系统自然降温, 此阶段 $Q_{\mathrm{p}}+Q_{\mathrm{w}}=0$, 公式 (7) 可推导为:

$$
t=-\frac{\sum_{i} m_{i} C_{i}}{h S} \ln \theta=-\tau_{\mathrm{s}} \ln \theta
$$

$\tau_{\mathrm{s}}$ 为系统的时间常量, 表示时间 $t$ 与 $-\ln \theta$ 的直 线斜率。考虑到悬浮液中碳球质量为 $0.02 \mathrm{mg}$, 远小 于水的质量 $(0.2 \mathrm{~g})$, 其次, 水的比热容大于碳球 的比热容, 因此可以忽略 $m_{\mathrm{p}} C_{\mathrm{p}}$ 。由系统自然降温阶 段 $t$ 与- $\ln \theta$ 的线性关系，可求出 $h S$ :

$$
h S=\frac{m_{\mathrm{w}} C_{\mathrm{w}}}{\tau_{\mathrm{s}}}
$$

公式（5）中的 $Q_{\mathrm{w}}$ 可以通过近红外光照射纯水 的升温过程求得, 公式如下:

$$
Q_{\mathrm{w}}=\frac{m_{\mathrm{w}} C_{\mathrm{w}} \Delta T_{\max }}{\Delta t}
$$

式中, $m_{\mathrm{w}}$ 为水的质量, $C_{\mathrm{w}}$ 为水的比热容, $\Delta T_{\text {max }}$ 为 近红外光照射纯水达到系统平衡后的温度变化, $\Delta t$ 为近红外光的照射时长。

因此, 根据公式 (5) 和 (8 10) 即可计算出碳 球的光热转换效率 $\eta$ 。

\section{5 细胞生物相容性测试}

$\mathrm{HaCaT}$ 细胞 (人永生化表皮细胞, $2 \times 10^{4}$ 个/孔) 接种于 96 孔板中，培养 $12 \mathrm{~h}$ 后，磷酸盐缓冲液润 洗细胞, 加入 $100 \mu \mathrm{L}$ 含不同浓度碳球的新鲜培养基 分别培养 24 和 $48 \mathrm{~h}$, 使用 CCK-8 法测定细胞存活 率。

\section{6 肿瘤细胞光热效果表征}

MG-63 肿瘤细胞 (人骨肉瘤细胞, $2 \times 10^{4}$ 个/孔) 接种于 96 孔板中, 培养 $12 \mathrm{~h}$ 后, 磷酸盐缓冲液润 洗细胞, 加入 $100 \mu \mathrm{L}$ 含 $100 \mu \mathrm{g} / \mathrm{mL}$ 碳球的新鲜培养 基, 用 $0.8 \mathrm{~W} / \mathrm{cm}^{2}$ 近红外光照射肿瘤细胞 5 和 10 $\min$, 培养 $4 \mathrm{~h}$ 后, 使用 CCK-8 法测定各组细胞存 活率。使用 Calcein-AM 和 PI 对光热治疗后的细胞 进行染色，使用苂光显微镜观察细胞活性。

\section{2 结果与讨论}

\section{1 碳球的制备和表征}

采用超声辅助法可以促进邻苯三酚和甲酫分子 之间的聚合，实现 $5 \mathrm{~min}$ 内快速制备单分散的酚醛 树脂微球。制备流程如图 1 所示, 邻苯三酚和甲醛 在碱性环境下可以发生聚合, 形成体式酚醛树脂, 但反应时间较长。溶液经超声处理, 加快了分子间 的聚合，使反应速率迅速提升，生成的酚醛树脂微 球经炦烧处理即可得到单分散、粒径均一的碳球。

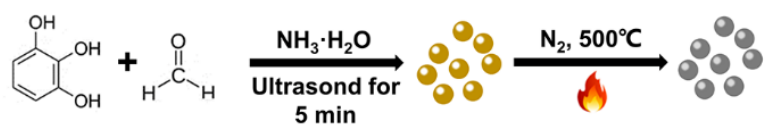

图 1 碳球制备示意图

Fig. 1 Formation process of the carbon spheres

改变邻苯三酚和甲醛的反应比例, 可以对微球 的直径进行调控。图 2 为不同摩尔比的邻苯三酚与 甲醛所制备的微球，均有良好的单分散性。利用扫 
描电镜照片随机选取 100 个微球，对不同条件下合 成的微球直径进行测量和统计, 统计结果如图 3 所 示。邻苯三酚与甲醛的摩尔比由 $2: 1$ 变为 $1: 4$, 对应 的微球平均直径分别为 238、375、454 和 $367 \mathrm{~nm}$, 呈现先增大后减小的趋势。当甲醛参与反应的摩尔 比逐渐增大时, 分子间不断聚合, 使微球直径逐渐 增大。但摩尔比增大到一定程度后, 由于溶液中初 始聚合反应的位点剧烈增多, 限制了微球直径增大。
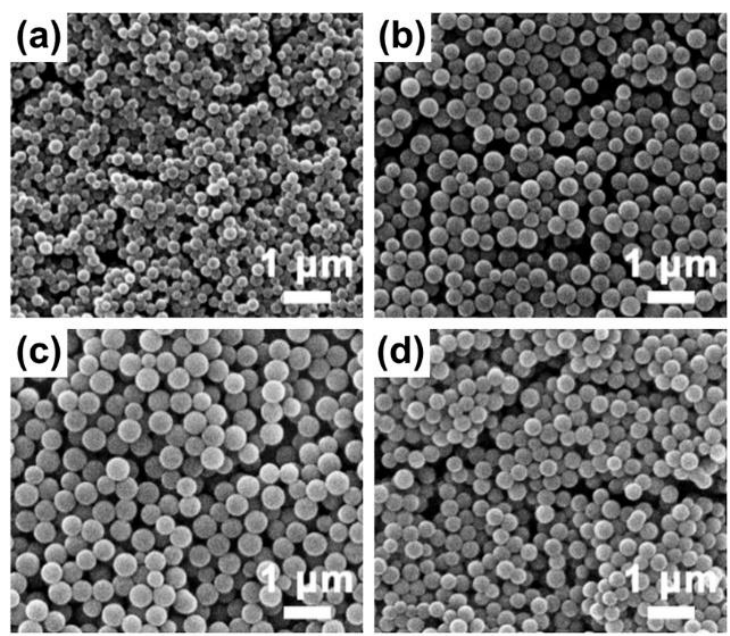

图 2 邻苯三酚与甲醛摩尔比为（a） 2:1、（b） $1: 1$ 、（c） $1: 2$ 和 (d) 1:4 时对酚醛树脂微球形貌的影响

Fig. 2 Effects of molar ratios of pyrogallic acid to formaldehyde at (a) $2: 1$, (b) $1: 1$, (c) 1:2, and (d) 1:4 on morphologies of phenolic resin spheres (a)

(c)

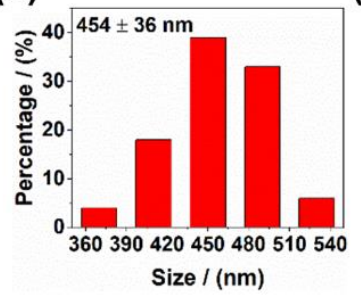

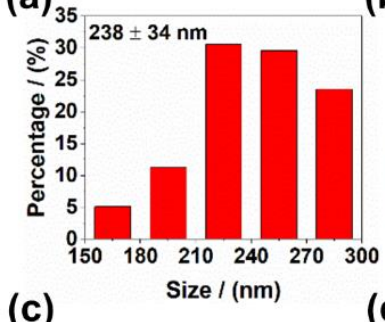

(b)

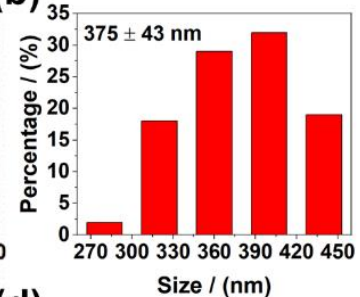

(d)

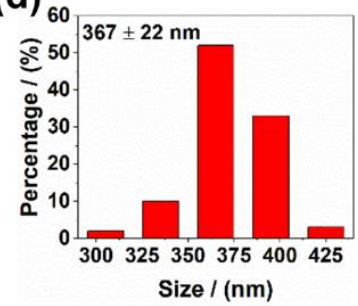

图 3 邻苯三酚与甲醛摩尔比为（a） $2: 1$ 、（b） $1: 1$ 、（c） $1: 2$ 和 (d) 1:4 时对酚醛树脂微球直径的影响

Fig. 3 Effects of molar ratios of pyrogallol to formaldehyde at (a) 2:1, (b) 1:1, (c) 1:2, and (d) 1:4 on particle sizes of phenolic resin spheres

将邻苯三酚和甲醛摩尔比为 $1: 2$ 条件下合成的

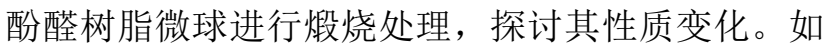
图 4(a,b)所示, 炦烧后, 碳球仍保持规则的球形,
并具有良好的单分散性。如图 4(c)所示, 动态光散 射分析结果表明经煅烧处理后，微球直径由 $492 \mathrm{~nm}$ 减小到 $341 \mathrm{~nm}$, 这主要是由于高温使酚醛树脂微球 发生热解和碳化 ${ }^{[27]}$, 从而导致直径减小。使用热重 分析对微球的碳化过程进行表征（图 4(d)），结果 表明随䍩烧温度的升高, 微球残留质量百分比逐渐 下降。当煅烧温度到达约 $700{ }^{\circ} \mathrm{C}$ 时, 微球质量基本 保持稳定, 质量百分比约为 $46 \%$ 。相比之下, 当微 球在 $500{ }^{\circ} \mathrm{C}$ 下瑖烧时, 其质量减少到 $58 \%$, 结果表 明在此条件下制备得到的碳球已具有较高的碳化程 度。
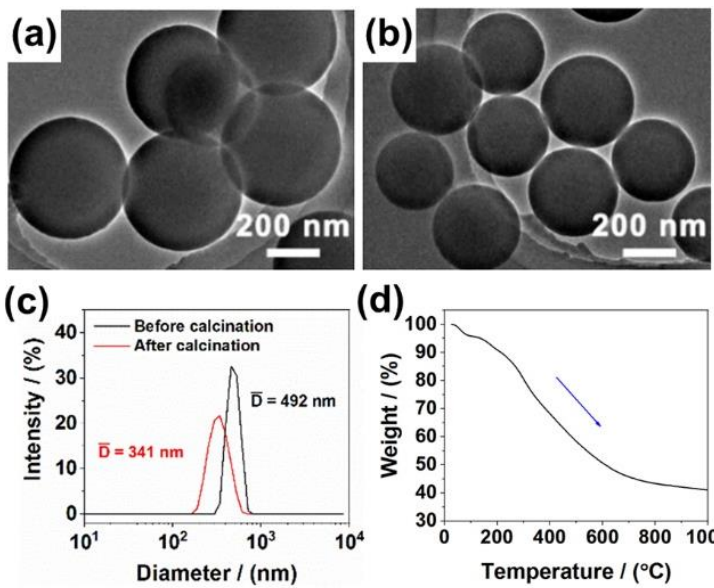

(d)

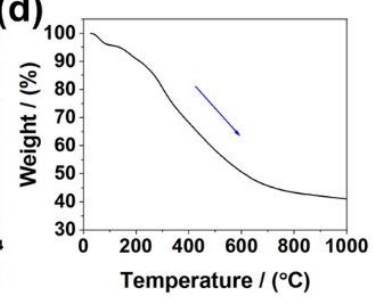

图 4 酚醛树脂微球（a）煅烧前与（b）耘烧后的 TEM 照片 及其 (c) 动态光散射粒度分析曲线和 (d) 炦烧后的碳球在 $\mathrm{N}_{2}$ 气氛下的热重分析曲线

Fig. 4 TEM morphologies of phenolic resin spheres (a) before and (b) after calcination, their (c) particle size analysis curves of dynamic light scattering and (d) thermogravimetric analysis curve of carbon spheres after calcination in $\mathrm{N}_{2}$ atmosphere

图 5(a)为傅里叶红外光谱分析微球的结果。 $3430 \mathrm{~cm}^{-1}$ 为羟基- $\mathrm{OH}$ 的伸缩振动峰, 代表微球表面 的酚羟基以及吸附的水分子 ${ }^{[28] ； 1481 ~ 1624 ~} \mathrm{~cm}^{-1}$ 代 表芳香环上 $\mathrm{C}=\mathrm{C}$ 和 $\mathrm{C}-\mathrm{C}$ 的伸缩振动峰; $1380 \mathrm{~cm}^{-1}$ 为酚醛基团平面内 $\mathrm{O}-\mathrm{H}$ 的弯曲振动峰, 代表分子内 部的酚着基 ${ }^{[29-30]} ; 1118 \mathrm{~cm}^{-1}$ 为 C-O-C 的伸缩振动峰, 与邻苯三酚和甲醛的聚合反应产生的醚键有关 ${ }^{[31]}$ 。 与酚醛树脂微球相比, 经过羟烧处理后, 微球表面 的官能团减少, 特征峰 1118 和 $1380 \mathrm{~cm}^{-1}$ 消失。这 是因为高温煅烧使酚醛树脂发生热解, 含氧官能团 被逐渐脱除。但衤烧处理后的微球表面仍含有部分 亲水官能团, 如羟基 ( $-\mathrm{OH})$, 这有利于碳球在水中 的分散。

图 5(b)为拉曼光谱图, 包含 $D$ 峰和 $G$ 峰两个特 征峰, 分别位于 1351 和 $1586 \mathrm{~cm}^{-1}$ 。D 峰代表碳材 
料结构的无序度和内部结构缺陷, $\mathrm{G}$ 峰代表碳原子 的 $\mathrm{sp}^{2}$ 杂化，体现碳材料的石墨化程度。通过计算 $\mathrm{D}$ 峰与 $\mathrm{G}$ 峰强度的比值 $I_{\mathrm{D}} / I_{\mathrm{G}}$ 可以比较陆烧前后微球 内部碳结构的变化 ${ }^{[32]}$ 。煅烧前, 酚醛树脂微球 $I_{\mathrm{D}} / I_{\mathrm{G}}$ 为 0.69 , 煅烧后, $I_{\mathrm{D}} / I_{\mathrm{G}}$ 增加到 0.89 , 这说明高温煅 烧处理增加了碳层的无序化程度和内部缺陷, 这与 之前的研究相吻合 ${ }^{[33]}$, 其缺陷的存在对于碳球的性 质具有重要的影响。
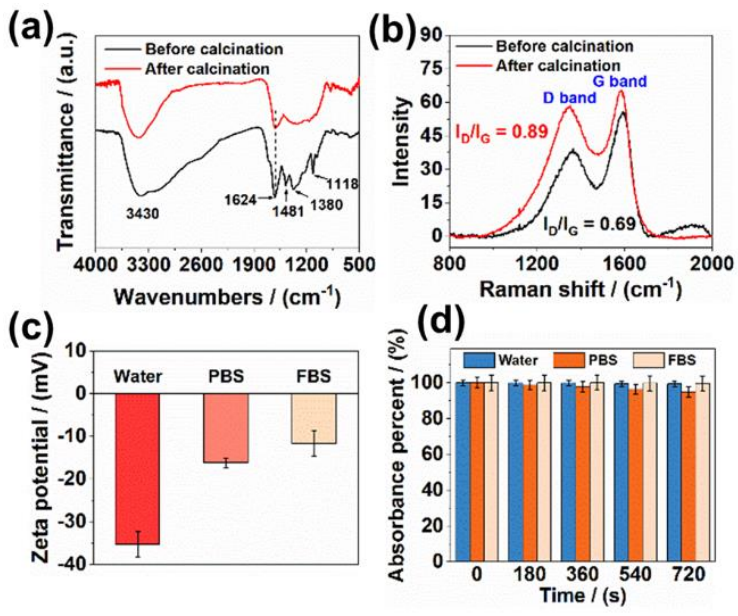

图 5 微球煅烧前后的（a）傅里叶红外光谱图和（b）拉曼光 谱图及其（c）碳球分散到不同介质中的 Zeta 电位和（d）在 $808 \mathrm{~nm}$ 处吸光度随时间变化的百分比

Fig. 5 (a) FT-IR and (b) Raman spectra of phenolic resin spheres before and after calcination, (c) Zeta potential of carbon spheres in different dispersion medium and (d) absorbance percent of carbon spheres in different dispersion medium at $808 \mathrm{~nm}$

为了验证碳球在不同介质中的分散稳定性, 本 实验分别使用纯水、磷酸盐缓冲液 (PBS) 和胎牛血 清 (FBS) 分散碳球。图 5(c) 为碳球在上述三种介质 中的 Zeta 电位测试结果, 在三种介质中碳球的电位 均为负值，这是由于碳球表面带有大量负电性的官 能团 (比如-OH), 这与傅里叶红外光谱结果相吻合, 羟基有利于碳球在水中稳定。其中，碳球在纯水中 的电位最高, 约为 $-35.2 \mathrm{mV}$, 在 PBS 和 FBS 中有所 降低, 分别为 -16.2 和 $-11.6 \mathrm{mV}$ 。在上述三种介质中, 液相中的正电荷离子吸附到带负电荷的碳球表面, 在两相界面呈扩散状态分布形成了双电层。相比于 纯水, 由于后两种生理介质中存在大量的正电荷 (PBS 中存在 $\mathrm{Na}^{+}$和 $\mathrm{K}^{+}$等, FBS 中存在带正电的蛋 白等），这些带正电荷的离子与碳球表面的负电荷 作用, 使双电层结构发生变化, 导致碳球的 Zeta 电 位降低。图 5(d)为碳球分散到上述三种介质中随时 间变化的吸光度百分比, 结果表明碳球在三种介质 中均具有良好的分散稳定性。随时间变化, 碳球在 纯水和 FBS 中的稳定性较好, 在 PBS 中的吸光度
有所减小, 但变化小于 $5 \%$ 。以上结果说明碳球在生 理介质中，具有良好的分散稳定性，有利于扩展其 生物应用。

\section{2 碳球的光热性能}

图 6(a) 为碳球悬浮液在不同近红外光强度 $(0.2$ 、 0.6 和 $\left.1.0 \mathrm{~W} / \mathrm{cm}^{2}\right)$ 照射下的升温曲线。经过 $10 \mathrm{~min}$ 照射后, 悬浮液的温度分别升高了 $8.2 、 18.3$ 和 $30.8{ }^{\circ} \mathrm{C}$ 。图 6(b) 为不同浓度的碳球悬浮液 ( 0 、50、 100、和 $200 \mu \mathrm{g} / \mathrm{mL}$ ）在 $0.8 \mathrm{~W} / \mathrm{cm}^{2}$ 近红外光照射下 的温度曲线。结果表明碳球悬浮液具有明显的光热 效应，随碳球浓度增加，悬浮液分别升温 16.3、27.3 和 $35.9{ }^{\circ} \mathrm{C}$ 。作为对照组, 纯水仅升温 $0.4{ }^{\circ} \mathrm{C}$, 说明 纯水对近红外光无明显吸收。图 6(c)表明碳球能够 高效地吸收近红外光并将其转换为热量, 使周围的 介质升温。光照 $4 \mathrm{~min}$ 后, 温度场分布均匀, 系统 温度达到平衡。为了验证碳球的光热稳定性, 用 0.8 $\mathrm{W} / \mathrm{cm}^{2}$ 的近红外光照射碳球悬浮液, 照射 $5 \mathrm{~min}$ 后, 关闭激光器, 使悬浮液自然冷却 $5 \mathrm{~min}$, 然后再打开 激光器, 使用相同强度的近红外光照射样品 4 个循 环。图 6(d)显示, 经过 4 次光热循环后, 碳球仍能 升温到稳定的温度, 说明碳球具有良好的光热稳定 性，有利于肿瘤的重复光热治疗应用。

碳球具有良好的光热性能, 通过监测光热转换 过程, 可以计算光热转换效率。如图 7(a,b)所示, 近红外光照射碳球悬浮液 $8 \mathrm{~min}$ 后, 悬浮液温度升 高了 $24.1^{\circ} \mathrm{C}$ 。此时, 关闭激光器, 使系统自然冷却 降温, 根据降温曲线可得 $\tau_{\mathrm{s}}=180.46 \mathrm{~s}$, 从而计算出 $h S=0.0047 \mathrm{~W} /{ }^{\circ} \mathrm{C}$ 。如图 7(c) 所示, 悬浮液在近红外 区域具有连续的吸收, 在波长为 $808 \mathrm{~nm}$ 处的吸光度 为 0.4723 。纯水经近红外光照射转换的热量 $Q_{\mathrm{w}}=$ $0.0007 \mathrm{~W}$, 输入系统的近红外光功率 $I=0.41 \mathrm{~W}$, 将 相关参数代入公式 (5) 可计算得到碳球的光热转换 效率 $\eta=41.4 \%$, 此光热转换效率优于碳纳米管 (32\%) [34]、氧化石墨烯 (35\%) [22]和介孔碳球 (35.7\%) [35]。 这可能是因为经㷽烧处理后, 微球分子骨架迅速发 生热解和碳化, 而碳材料对近红外光具有连续较强 的吸收, 从而表现出优良的光热效应。其次, 高温 炦烧能够促使碳层内部形成大量的微小缺陷, 这些 缺陷位点具有较高的活性, 被激发的电子能够以缺 陷位点为中心发生非辐射复合，从而进一步提升碳 球的光热转换效率 $[36-38]$ 。煅烧处理虽然能够影响碳 球的光热转换效率，但是从碳球的生物应用角度考 虑，期望其兼具良好的分散稳定性和光热性能。然 而, 碳球经高温炦烧处理后, 其含氧官能团会被迅 
速脱除，耘烧温度越高，碳球的碳化程度越高，含 氧官能团脱除得越彻底，这会导致碳球在生理质中 难以稳定分散，从而限制其后续的生物应用[39]。结 合热重分析和傅里叶红外光谱结果, 本研究在 $500^{\circ} \mathrm{C}$

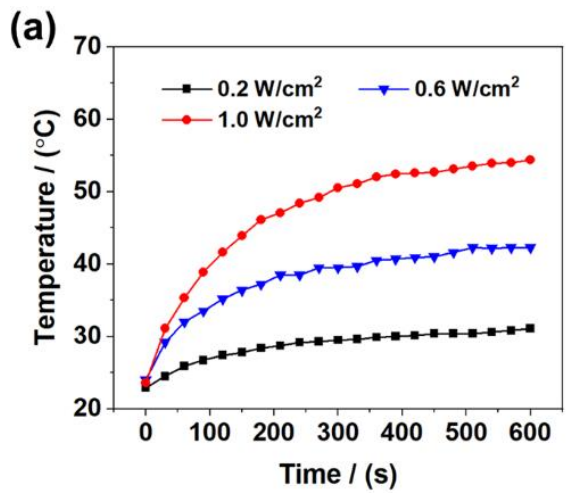

(c)

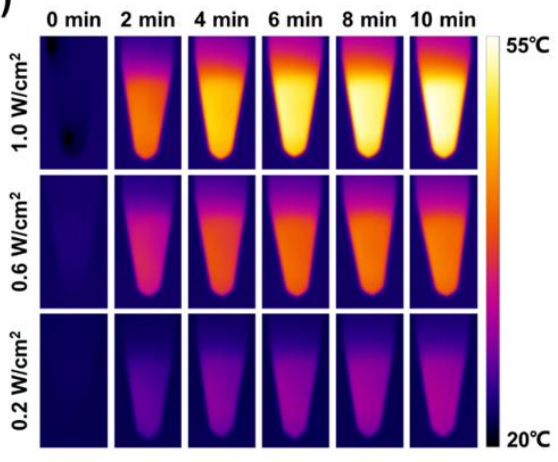

下耘烧处理所制备的碳球，既能确保烣烧后应具有 的良好分散稳定性，确保其能够稳定分散于生理介 质中，同时又保证了碳球的光热转换效率能维持在 较高的水平，从而实现高效的光热治疗。

(b)

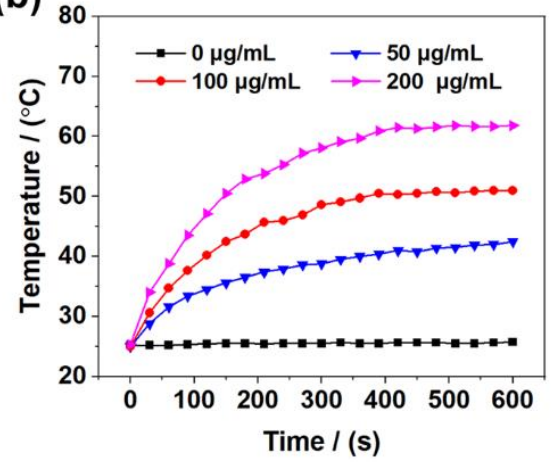

(d)

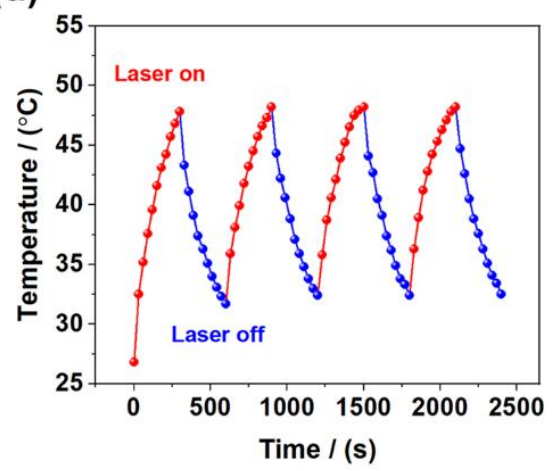

图 6 碳球的光热性能测试

Fig. 6 Evaluation of photothermal effect of carbon spheres

Influence of (a) laser power density (concentration: $100 \mu \mathrm{g} / \mathrm{mL}$ ) and (b) concentration of carbon spheres (laser power density: $0.8 \mathrm{~W} / \mathrm{cm}^{2}$ ) on photothermal effect; (c) Cloud images of temperature changes; (d) Measurement of the photothermal stability (concentration: $100 \mu \mathrm{g} / \mathrm{mL}$, laser power density: $0.8 \mathrm{~W} / \mathrm{cm}^{2}$ )

(a)

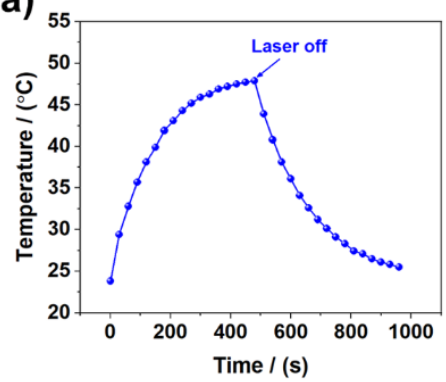

(b)

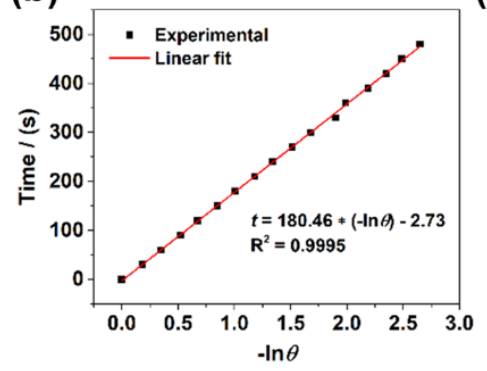

(c)

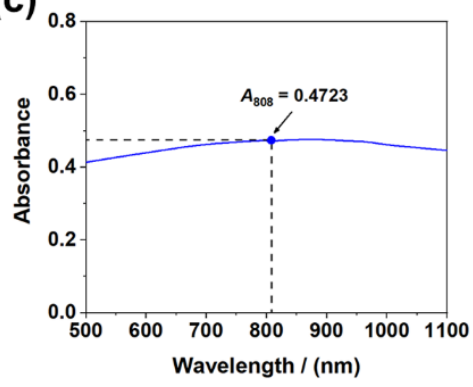

图 7 碳球的光热转换效率测试

Fig. 7 Measurement of the photothermal conversion efficiency of carbon spheres

(a) Heating and cooling curves of carbon sphere suspension (concentration: $100 \mu \mathrm{g} / \mathrm{mL}$, laser power density: $0.8 \mathrm{~W} / \mathrm{cm}^{2}$ ); (b) Linear fitting curve of $t$ and $-\ln \theta$ at cooling phase; (c) UV-visible absorption curve of carbon sphere suspension

\section{3 碳球的细胞生物相容性}

用 $\mathrm{HaCaT}$ 细胞作为碳球细胞相容性测试的正 常人体模型细胞, 将不同浓度的碳球与细胞共培养, 检测细胞的存活率。图 8 结果表明，不同浓度的碳
球（25、50、100 和 $200 \mu \mathrm{g} / \mathrm{mL}$ ) 在与细胞共培养 24 和 $48 \mathrm{~h}$ 后, 细胞的存活率仍高于 $94 \%$, 证明碳球的 细胞毒性较低, 具有良好的细胞生物相容性, 适用 于生物应用。 


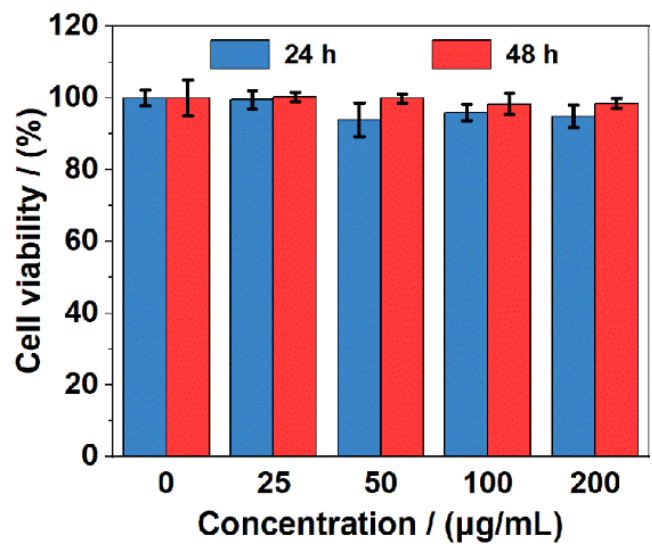

图 8 不同浓度的碳球与 $\mathrm{HaCaT}$ 细胞共培养 24 和 $48 \mathrm{~h}$ 后的 细胞存活率

Fig. 8 Viability of $\mathrm{HaCaT}$ cells co-cultured with different concentrations of carbon spheres for 24 and $48 \mathrm{~h}$

\section{4 碳球对肿瘤细胞的光热杀伤效果}

用 MG-63 骨肿瘤细胞作为碳球光热治疗的模 型细胞, 测定不同光热时长对细胞存活率的影响。 图 9 表明, 当近红外光照射含碳球的肿瘤细胞时, 随着光照时长的延长, 细胞活性显著降低。照射 5 和 $10 \mathrm{~min}$ 近红外光的细胞存活率分别下降到 52.6\% 和 $15.9 \%$ 。与此相比, 近红外光照射不含碳球的肿 瘤细胞时, 细胞存活率无明显变化, 说明单独照射 近红外光而无碳球的作用不会诱导细胞死亡。

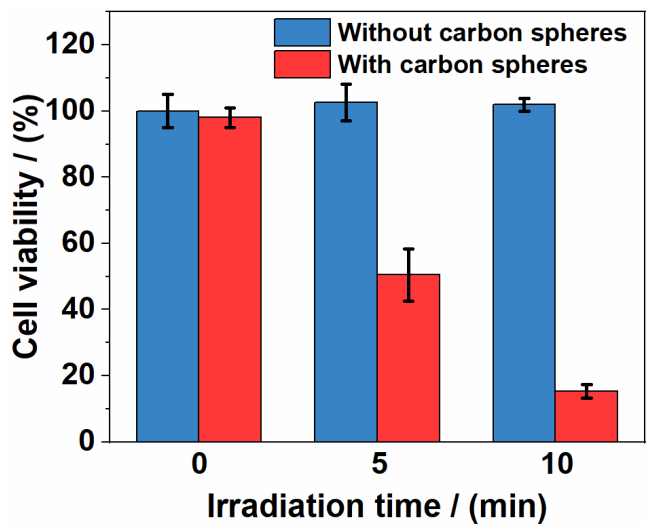

图 9 碳球在近红外光照射不同时长处理时对肿瘤细胞的光 热杀伤效果

Fig. 9 Photothermal killing effect of carbon spheres on tumor cells with different laser irradiation time

肿瘤细胞经光热治疗后, 荧光染色结果如图 10 所示。未被照射近红外光时，肿瘤细胞表现出明显 的活细胞绿色苂光, 表明细胞几乎没有死亡。当近 红外光分别照射与碳球共培养的肿瘤细胞 5 和 10 $\min$ 后, 结果显示红色荧光的死细胞逐渐增多, 绿 色苂光的活细胞显著减少。表明经过光热治疗后,
肿瘤细胞大量死亡, 随光热时长的延长, 杀伤肿瘤 细胞效果显著增强。结果证明了所制备的碳球可以 高效地诱导光热效应, 有效杀伤肿瘤细胞。

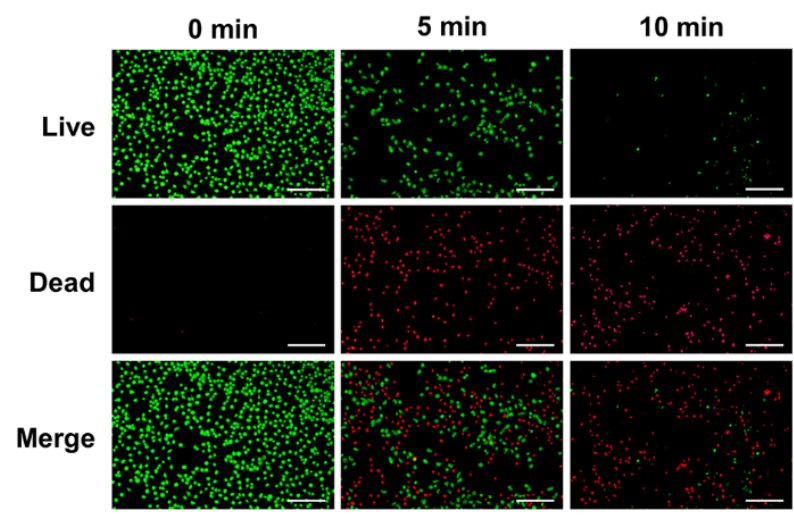

图 10 含碳球的肿瘤细胞悬液经不同时长近红外光照射后的 活、死细胞苂光照片

Fig. 10 Live/dead fluorescence images of tumor cells co-culcured with carbon spheres with different laser irradiation time (Green: live cells; Red: dead cells; Scale bar=200 $\mu \mathrm{m}$ )

\section{3 结论}

本研究基于超声辅助法快速制备了一种单分散 碳球, 并将其用于肿瘤细胞光热治疗。该方法可以实 现邻苯三酚和甲醛的快速聚合, $5 \mathrm{~min}$ 内合成酚醛树 脂微球, 经 $500{ }^{\circ} \mathrm{C}$ 㷽烧处理得到碳球, 制备工艺简单。 所制备的碳球在近红外区具有连续波长的吸收，在 $808 \mathrm{~nm}$ 的近红外光照射下，具有良好的光热效应和 光热稳定性, 光热转换效率达到 $41.4 \%$ 。而且, 该碳 球具有良好的细胞生物相容性, 能够有效地杀伤肿 瘤细胞。综上, 所制备的碳球有望应用于肿瘤光热治 疗。

\section{参考文献:}

[1] SCHROEDER A, HELLER D A, WINSLOW M M, et al. Treating metastatic cancer with nanotechnology. Nature Reviews. Cancer, 2012, 12(1): 39-50.

[2] LUETKE A, MEYERS P A, LEWIS I, et al. Osteosarcoma treatment - where do we stand? A state of the art review. Cancer Treatment Reviews, 2014, 40(4): 523-532.

[3] ZOU L L, WANG H, HE B, et al. Current approaches of photothermal therapy in treating cancer metastasis with nanotherapeutics. Theranostics, 2016, 6(6): 762-772.

[4] ZHI D F, YANG T, O'HAGAN J, et al. Photothermal therapy. Journal of Controlled Release, 2020, 325: 52-71.

[5] LIU S, PAN X T, LIU H Y. Two-dimensional nanomaterials for photothermal therapy. Angewandte Chemie International Edition, 2020, 59(15): 5890-5900.

[6] KIM H, CHUNG K, LEE S, et al. Near-infrared light- 
responsive nanomaterials for cancer theranostics. Wiley Interdisciplinary Reviews-Nanomedicine and Nanobiotechnology, 2016, 8(1): 23-45.

[7] JAQUE D, MARTINEZ MAESTRO L, DEL ROSAL B, et al. Nanoparticles for photothermal therapies. Nanoscale, 2014, 6(16): 9494-9530.

[8] PAN H Y, ZHANG C, WANG T T, et al. In situ fabrication of intelligent photothermal indocyanine green-alginate hydrogel for localized tumor ablation. ACS Applied Materials \& Interfaces, 2019, 11(3): 2782-2789.

[9] LIU B, SUN J, ZHU J J, et al. Injectable and NIR-responsive DNA-inorganic hybrid hydrogels with outstanding photothermal therapy. Advanced Materials, 2020, 32(39): 2004460.

[10] FERNANDES N, RODRIGUES C F, MOREIRA A F, et al. Overview of the application of inorganic nanomaterials in cancer photothermal therapy. Biomaterials Science, 2020, 8(11): 29903020 .

[11] SONG X J, CHEN Q, LIU Z. Recent advances in the development of organic photothermal nano-agents. Nano Research, 2014, 8(2): 340-354.

[12] JIN R M, YANG J, ZHAO D H, et al. Hollow gold nanoshells-incorporated injectable genetically engineered hydrogel for sustained chemo-photothermal therapy of tumor. Journal of Nanobiotechnology, 2019, 17(1): 99.

[13] ZHU X M, WAN H Y, JIA H L, et al. Porous Pt nanoparticles with high near-infrared photothermal conversion efficiencies for photothermal therapy. Advanced Healthcare Materials, 2016, 5(24): 3165-3172.

[14] WENG Y Z W, GUAN S Y, WANG L, et al. Hollow carbon nanospheres derived from biomass by-product okara for imaging-guided photothermal therapy of cancers. Journal of Materials Chemistry B, 2019, 7(11): 1920-1925.

[15] LIU W, ZHANG X Y, ZHOU L, et al. Reduced graphene oxide (rGO) hybridized hydrogel as a near-infrared (NIR)/pH dual-responsive platform for combined chemo-photothermal therapy. Journal of Colloid and Interface Science, 2019, 536: 160-170.

[16] WANG J X, YAO C J, SHEN B, et al. Upconversionmagnetic carbon sphere for near infrared light-triggered bioimaging and photothermal therapy. Theranostics, 2019, 9(2): 608-619.

[17] XIE H H, LI Z B, SUN Z B, et al. Metabolizable ultrathin $\mathrm{Bi}_{2} \mathrm{Se}_{3}$ nanosheets in imaging-guided photothermal therapy. Small, 2016, 12(30): 4136-4145.

[18] JIANG G B, HUA Q, WEN S W, et al. Carbon dot/WS heterojunctions for NIR-II enhanced photothermal therapy of osteosarcoma and bone regeneration. Chemical Engineering Journal, 2020, 383: 123102.

[19] DONG L L, JI G M, LIU Y, et al. Multifunctional Cu- $\mathrm{Ag}_{2} \mathrm{~S}$ nanoparticles with high photothermal conversion efficiency for photoacoustic imaging-guided photothermal therapy in vivo. Nanoscale, 2018, 10(2): 825-831.

[20] NEELGUND G M, OKI A R. Influence of carbon nanotubes and graphene nanosheets on photothermal effect of hydroxyapatite. Journal of Colloid and Interface Science, 2016, 484: $135-145$.

[21] ZHANG M, WANG W T, WU F, et al. Magnetic and fluorescent carbon nanotubes for dual modal imaging and photothermal and chemo-therapy of cancer cells in living mice. Carbon, 2017, 123: 70-83.

[22] LI P, YAN Y, CHEN B L, et al. Lanthanide-doped upconversion nanoparticles complexed with nano-oxide graphene used for upconversion fluorescence imaging and photothermal therapy. Biomaterials Science, 2018, 6(4): 877884.
[23] LIU H J, LI C W, QIAN Y, et al. Magnetic-induced graphene quantum dots for imaging-guided photothermal therapy in the second near-infrared window. Biomaterials, 2020, 232: 119700 .

[24] ZHOU L, DONG K, CHEN Z W, et al. Near-infrared absorbing mesoporous carbon nanoparticle as an intelligent drug carrier for dual-triggered synergistic cancer therapy. Carbon, 2015, 82: 479-488.

[25] GE J C, JIA Q Y, LIU W M, et al. Red-emissive carbon dots for fluorescent, photoacoustic, and thermal theranostics in living mice. Advanced Materials, 2015, 27(28): 4169-4177.

[26] LIU Y L, AI K L, LIU J H, et al. Dopamine-melanin colloidal nanospheres: an efficient near-infrared photothermal therapeutic agent for in vivo cancer therapy. Advanced Materials 2013, 25(9): 1353-1359.

[27] WANG S J, WANG Y, BIAN C, et al. The thermal stability and pyrolysis mechanism of boron-containing phenolic resins: the effect of phenyl borates on the char formation. Applied Surface Science, 2015, 331: 519-529.

[28] POL V G, SHRESTHA L K, ARIGA K. Tunable, functional carbon spheres derived from rapid synthesis of resorcinolformaldehyde resins. ACS Applied Materials \& Interfaces, 2014, 6(13): 10649-10655.

[29] ZHAO X, ZHANG M, SUN X D, et al. Comprehensive understanding of the formation process on monodisperse resorcinol-formaldehyde polymer and carbon spheres and their use as substrates for surface-enhanced Raman spectroscopy. Applied Surface Science, 2020, 506: 144591.

[30] CAN M, BULUT E, ÖZACAR M. Synthesis and characterization of pyrogallol-formaldehyde nano resin and its usage as an adsorbent. Journal of Chemical and Engineering Data, 2012, 57(10): 2710-2717.

[31] LIU M M, CAI C, LI J, et al. Stober synthesis of tannic acid-formaldehyde resin polymer spheres and their derived carbon nanospheres and nanocomposites for oxygen reduction reaction. Journal of Colloid and Interface Science, 2018, 528: $1-9$.

[32] GUAN Q, ZHOU L L, ZHOU L N, et al. A carbon nanomaterial derived from a nanoscale covalent organic framework for photothermal therapy in the NIR-II biowindow. Chemical Communications, 2020, 56: 7793-7796.

[33] LIANG Z G, XIA H, ZHANG L M, et al. One-pot synthesis of monodisperse phenolic resin spheres with high thermal stability and its derived carbon spheres as supercapacitor electrodes. Results in Physics, 2020, 16: 102912.

[34] NEELGUND G M, OKI A. Advancement in photothermal effect of carbon nanotubes by grafting of poly(amidoamine) and deposition of CdS nanocrystallites. Industrial \& Engineering Chemistry Research, 2018, 57(23): 7826-7833.

[35] ZHOU L B, JING Y, LIU Y B, et al. Mesoporous carbon nanospheres as a multifunctional carrier for cancer theranostics. Theranostics, 2018, 8(3): 663-675.

[36] WENG Y Z W, GUAN S Y, WANG L, et al. Defective porous carbon polyhedra decorated with copper nanoparticles for enhanced NIR-driven photothermal cancer therapy. Small, 2020, 16(1): e1905184.

[37] ZHU J, MU S. Defect engineering in carbon-based electrocatalysts: insight into intrinsic carbon defects. Advanced Functional Materials, 2020, 30: 2001097.

[38] WANG W, SHANG L, CHANG G J, et al. Intrinsic carbondefect-driven electrocatalytic reduction of carbon dioxide. Advanced Materials, 2019, 31: 1808276.

[39] ZHAO P, ZHU L L. Dispersibility of carbon dots in aqueous and/or organic solvents. Chemical Communications, 2018, 54: 5401-5406. 